\title{
Correction of nonlinear distortion in high-transverse-emittance ratio-beam production with linear accelerator
}

\author{
Shaoheng Wang \\ LSU/CAMD, Louisiana State University, Center for Advanced Microstructures \& Devices, 6980 Jefferson Highway, \\ Baton Rouge, Louisiana 70806, USA \\ (Received 3 January 2008; published 2 May 2008)
}

\begin{abstract}
Derbenev proposed producing a high quality flat beam of high-transverse-emittance ratio (HTER) with a linear accelerator. Kim also discussed the round-to-flat transformation of angular-momentum-dominated beam. Fermilab/NICADD Photoinjector Laboratory has performed many experiments on HTER beam production. Experiments and simulations, collectively, showed an S-shaped transverse distribution in the flat beam. In this paper, the source of this emittance deterioration in the transformation is identified as the nonlinear rf cavity focusing force; and a solution is proposed.
\end{abstract}

PACS numbers: 29.27. $-\mathrm{a}, 41.85 .-\mathrm{p}$, 41.75.Fr, 41.75.Lx

\section{INTRODUCTION}

An electron beam with a high-transverse-emittance ratio (TER) has a flat cross section and maintains a flat shape while traveling along a beam line until coupling forces act on the beam. A flat beam consisting of high brightness and high TER is desired in several situations. A linear collider requires a flat beam to suppress beamstrahlung at the interaction point [1]. It is also needed in the proposed LBNL LUX project [2] and has potential application in the Smith-Purcell FEL [3].

A high quality, low-cost high-transverse-emittance ratio (HTER) beam could be produced with a photoinjector LINAC [4]. Burov [5] used circular basis to describe the coupled motion of electrons in a solenoid field and used planar basis to describe the decoupled motion following a round-to-flat transformation. The transformation is realized by diagonalization of the circular basis and implemented with a skew quadrupole triplet. Kim [6] simplified the theory by utilizing two invariants of the sigma matrix of round beam under symplectic transformation in 4D phase space. The discussion is limited to the linear transformation.

In the experiments as well as the simulations, the obtained HTER beam shows an S-shaped distribution, which deteriorates the emittance ratio. In this paper, it is shown that the nonlinear $\mathrm{rf}$ focusing force from the rf cavity is the source of the S-shaped distribution. With an extra solenoid placed before the rf cavity, the beam transverse size can be adjusted in such a way that the resulting nonlinear defocusing space charge force can be tuned to counteract the nonlinear $\mathrm{rf}$ focusing force. Simulations have shown that the S-shaped distribution can be straightened, and the emittance ratio can be enhanced.

\section{THE OBSERVATION OF S-SHAPED DISTRIBUTION IN HTER BEAM}

In the experimental setup of FNAL/A0 lab [7-9], a oneand-half-cell photo rf gun is immersed in a solenoid mag- netic field. The rf gun produces an angular-momentumdominated beam. After the beam travels out of the solenoid magnetic field region, it is accelerated to $17.5 \mathrm{MeV}$ by a TESLA-type 9-cell superconducting rf cavity. Then, the beam enters the round-to-flat-transformation region which is composed of three skew quadrupoles: the transformation adaptor. After the adaptor, a flat beam of TER $>50$ is obtained in experiments. Recently, TER is even enhanced to about 100 with $0.5 \mathrm{nC}$ bunch charge [10].

In this paper, PARMELA [11] is used to simulate the round-to-flat-transformation. Both experimental and simulation results are illustrated in Fig. 1. It can be observed that both results are not straight at the ends of the line distributions; the distributions are S-shaped. Of course, people can use only the center part of the flat beam by scrapping off the ends of the S-shaped line. But what if we want a HTER beam with a higher bunch charge? From the simulation result, it is apparent that the whole bunch occupies a nearly perfect elliptical area, and the majority of the particles center along the $\mathrm{S}$ line inside the ellipse. The TER of the beam can be greatly improved if we can
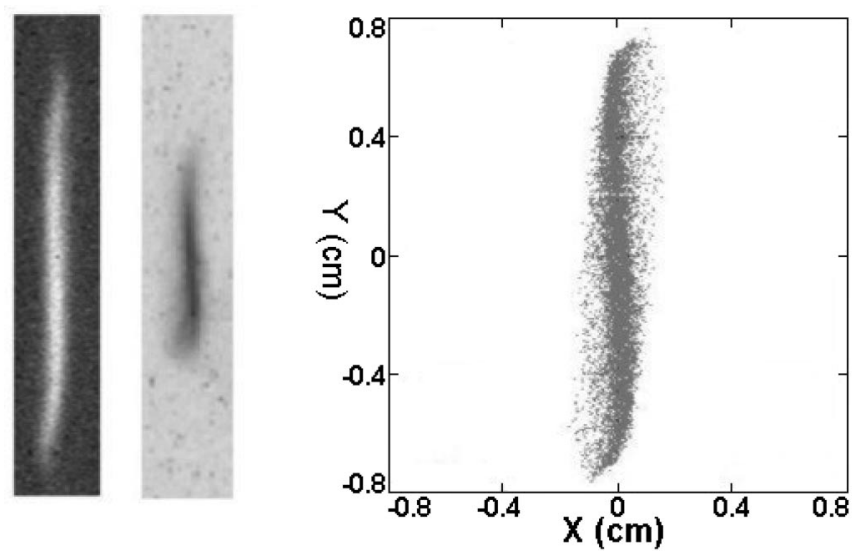

FIG. 1. Transverse distribution of the HTER beam. Left: two typical experiment results (courtesy of D. Edwards); right: PARMELA simulation result. 
make some compensation to get a straight line distribution in the final HTER beam.

\section{THE CONTRIBUTION FROM RF CAVITY'S NONLINEAR FOCUSING FORCE}

The S-shaped distribution looks like the well-known filamentation phenomenon which is the result of nonlinear forces. The difference is, this time it shows up in $X-Y$ space instead of $X-X^{\prime}$ and $Y-Y^{\prime}$ phase space.

But do not forget the round-to-flat transformation. During the transformation, the adaptor is supposed to remove the correlation between the motions in $X$ and $Y$ planes of the incoming angular-momentum-dominated round beam. The matching condition between the incoming beam and the adaptor is obtained by requiring the block diagonalization of the circular basis [5] or second-order beam moments matrix [6]. If we follow Kim's work, the round angular-momentum-dominated beam can be described by the $4 \mathrm{D}$ second-order moment matrix $\Sigma_{0}$ :

$$
\Sigma_{0}=\left(\begin{array}{cc}
\varepsilon_{\mathrm{eff}} T_{0} & \mathcal{L} J \\
-\mathcal{L} J & \varepsilon_{\mathrm{eff}} T_{0}
\end{array}\right)
$$

where

The quantity $\varepsilon_{\text {eff }}$ and $\beta$ can be interpreted as the emittance and the Courant-Snyder envelope function, respectively; $J$

$$
T_{0}=\left(\begin{array}{cc}
\beta & 0 \\
0 & \frac{1}{\beta}
\end{array}\right) .
$$

is the $2 \times 2$ unit symplectic matrix, and

$$
\mathcal{L}=\frac{1}{2}\left\langle x p_{y}-y p_{x}\right\rangle .
$$

Along the beam line, from the birth of the angularmomentum-dominated beam to the adaptor, the beam might experience some nonlinear focusing/defocusing forces. As a result of the nonlinear forces, the inner layer and outer layer of the beam see different transfer matrices. When the beam arrives at the adaptor, the values of $\beta$ along the radial direction become different. The matching condition of the round-to-flat transformation cannot be satisfied for all the layers of the beam simultaneously. Hence, following the transformation, the nonlinearity appears as the $\mathrm{S}$-shaped distribution in $X-Y$ space.

With the FNAL/A0 lab setup of the flat beam experiment, the transverse dimension of the round beam increases after it departs from the gun region. The transverse size of the beam reaches maximum when it enters the 9-cell cavity as evidenced by the PARMELA simulation result. The electron bunch is strongly focused at the entrance of the cavity. In the rf cavity, near the cell iris region, the nonlinear focusing force becomes more and more significant at radial locations further away from the cavity axis. A possible source of the S-shaped distribution is the nonlinear radial focusing in the 9-cell cavity.

To verify the contribution to the nonlinearity of the beam from the rf cavity, we set up a special simulation case. Before we proceed, we need to verify the ability to reach the matching condition of the adaptor in simulation. This is
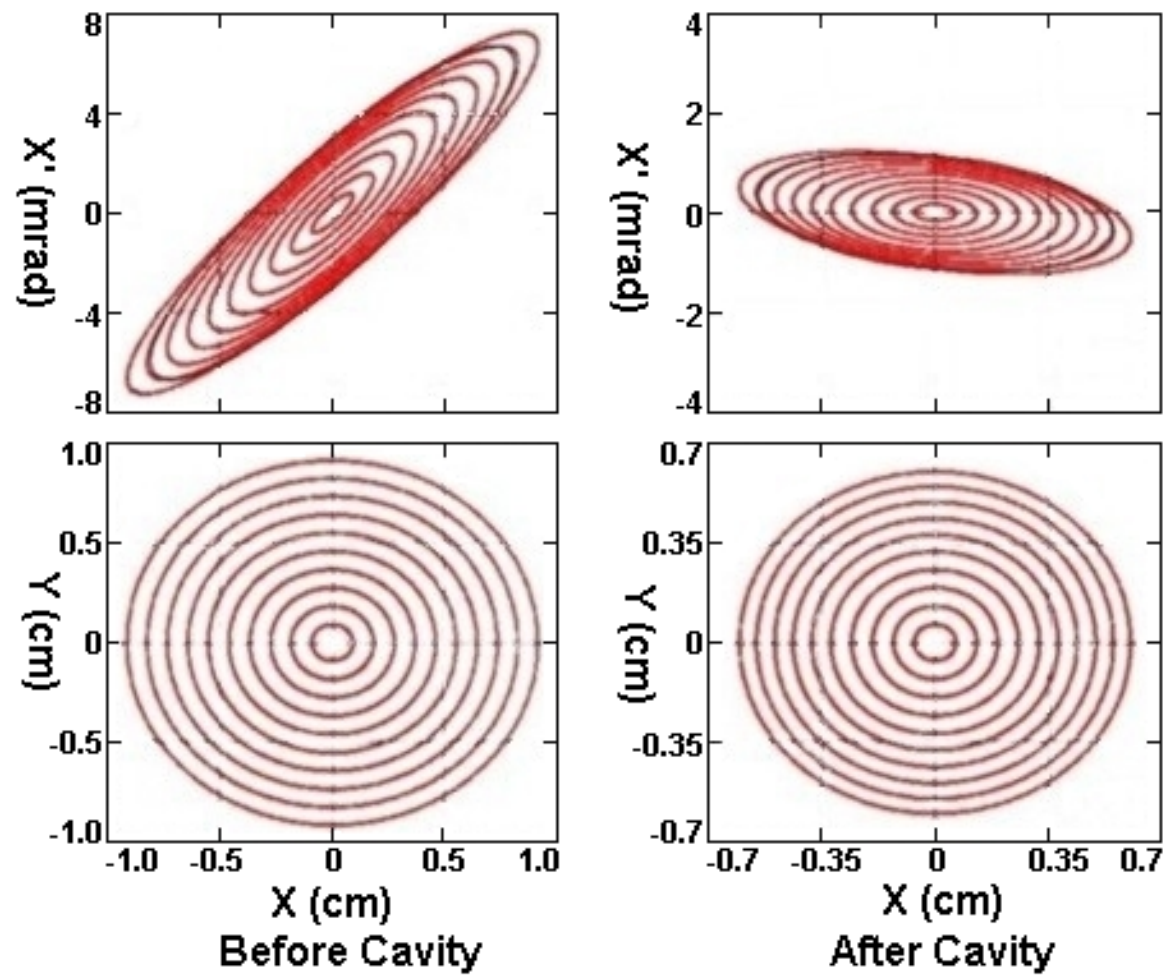

FIG. 2. (Color) Phase space structure before and after 9-cell rf cavity. 


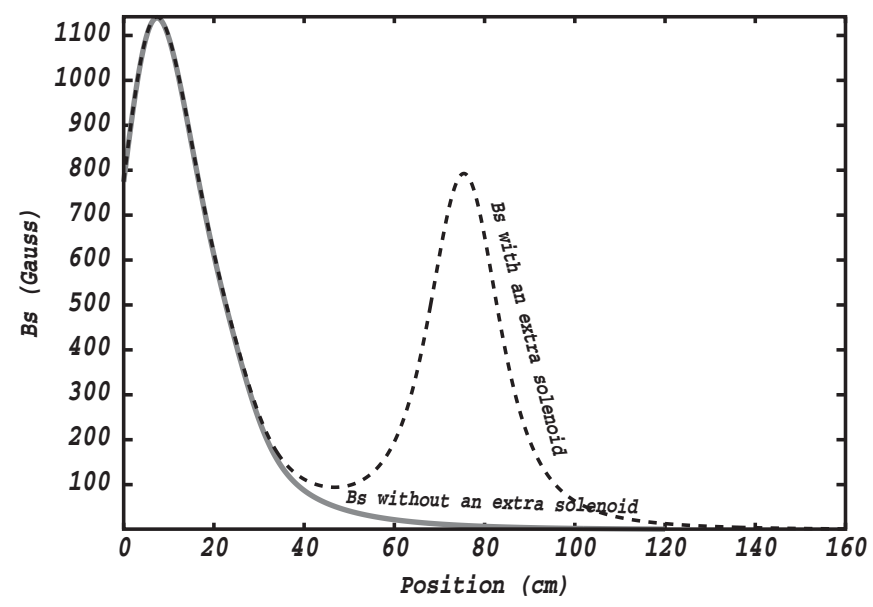

FIG. 4. $B_{s}$ profile for nonlinear correction.

provided with an angular-momentum-dominated round beam. Instead of letting it travel through the 9-cell cavity, the bunch directly passes through the adaptor. With space charge forces ignored, $\varepsilon_{y} / \varepsilon_{x}$ of the resulting flat beam can get as high as 21000 . Furthermore, in this ideal example, I can easily adjust the initial conditions of the bunch, such as the emittance, energy, and energy spread as well as transverse and longitudinal distribution. However, the S-shaped distribution did not show up.

Then, we tried to verify the rf cavity's contribution. Space charge force is ignored at this stage. For the bunch, we used a simplified model. Longitudinally, all the parti-

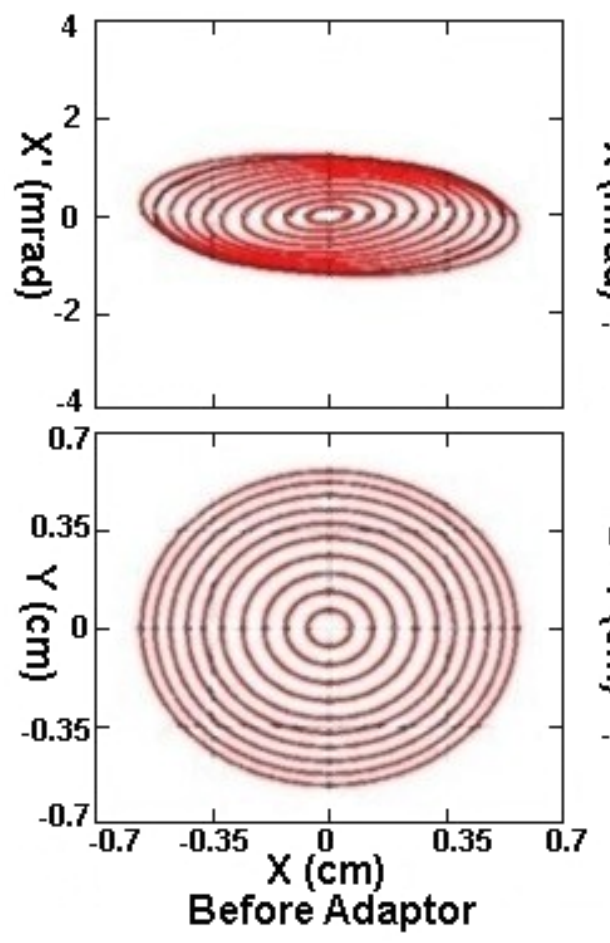

cles are distributed in one thin slice; transversely, all the particles are distributed in 10 radially evenly spaced circles of radii from 0.2 to $2 \mathrm{~mm}$. Of course, it is an angularmomentum-dominated beam. Then we let the beam of this simple distribution go through the 9-cell cavity. Let us compare the structures in phase space before and after the cavity. From the two upper graphs in Fig. 2, we can see that the outer ellipses in the phase space $\left(x, x^{\prime}\right)$ rotate slower than the inner ellipses. This phenomenon is hard to observe in $(x, y)$ space. It results from the nonlinear focusing force in rf cavity.

From there, we continue to pass the bunch through the adaptor. From Fig. 3, we see the deformation in $\left(x, x^{\prime}\right)$ and $\left(y, y^{\prime}\right)$ phase spaces before the adaptor has been mapped into $(x, y)$ and $\left(x^{\prime}, y^{\prime}\right)$ phase spaces after the adaptor. This is how the S-shaped distribution is established.

In the simulation result shown in Fig. 1, which includes the space charge force, the envelope of the whole bunch in $(x, y)$ space is a very good ellipse. We can see from both experimental and simulation results that most particles fall along the $\mathrm{S}$, and a small number of particles fill the remainder of the space in the ellipse. Therefore, the ellipse area is not very efficiently occupied. If we can straighten the $S$, then we can get a flat beam of smaller emittance.

\section{THE CORRECTION OF THE NONLINEAR DISTORTION}

Obviously, if we can decrease the transverse size of the bunch in the cavity, the bunch sees less nonlinear rf focus-

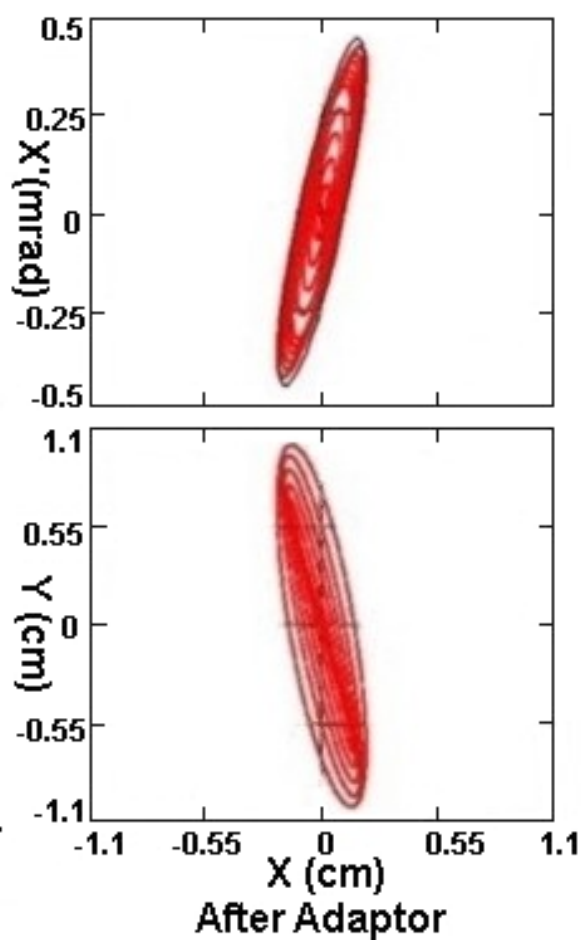

FIG. 3. (Color) Phase space structure before and after adaptor. 


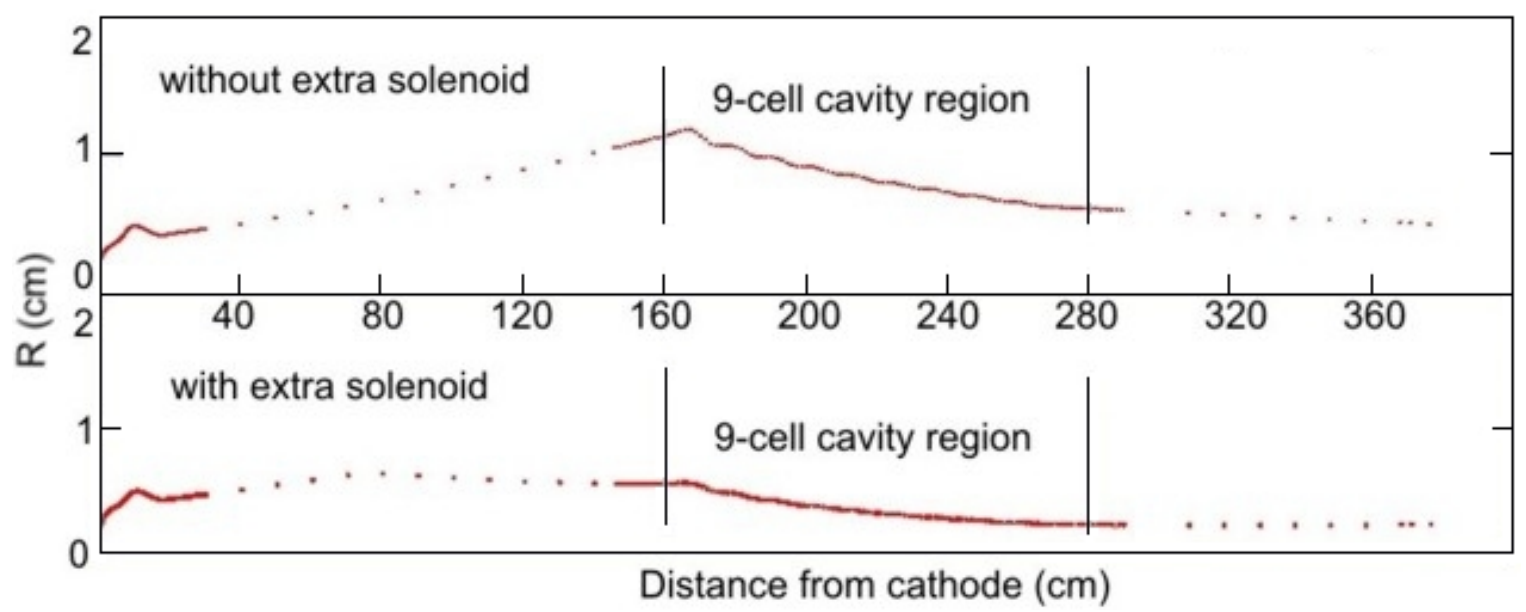

FIG. 5. (Color) Bunch radial profile for nonlinear correction.

ing force. Hence, the S-shaped distribution which results from the nonlinear effect will be less significant.

Furthermore, since the S-shaped distribution results from the nonlinear focusing force of the rf cavity, we might be able to cancel it to some extent by using some nonlinear defocusing force. A natural choice is nonlinear space charge force, which is defocusing. At the same time, we know that the nonlinear space charge force depends on the details of distribution and bunch shape. Hence, we can use the nonlinear defocusing space charge force to counteract the nonlinear rf focusing force if we are able to change the bunch shape and the distribution. In reality, this approach can be achieved by adding a solenoid before the cavity. On one hand, the solenoid provides a focusing effect, and the transverse size of the bunch will be smaller when it enters the cavity. This facilitates more particles moving away from the nonlinear $\mathrm{rf}$ focusing region. On the other hand, when the transverse size gets smaller in the cavity region, the nonlinear space charge force becomes stronger. This tunable nonlinear space charge force can offset the nonlinear rf focusing force. It should also be noted that all three forces, rf cavity focusing force, solenoid field focusing force, and space charge defocusing force, are axis symmetric. With this technique, we need to make sure the bunch is not overfocused by the solenoid. We know that we are pursuing a balance between the space charge force and the cavity rf focusing force to remove the nonlinear distortion by tuning the strength and position of the solenoid and the gradient and phase of the cavity. Attention should be paid to the proper radial profile within and after

TABLE I. Transformation results with and without nonlinear correction

\begin{tabular}{lcc}
\hline \hline Cases & $\varepsilon_{x}(\mu \mathrm{m})$ & $\varepsilon_{y}(\mu \mathrm{m})$ \\
\hline With correction & 0.529 & 45.87 \\
Without correction & 0.838 & 45.95 \\
\hline \hline
\end{tabular}

the cavity region (space charge force could be strong after the cavity).

Before the cavity, an extra solenoid is added while in simulation. Figure 4 shows the $B_{s}$ profile on the axis. The gray solid curve indicates the profile without the compensation solenoid and the dashed curve with the compensation solenoid. Note that $B_{s} \neq 0$ at cathode plane which is required for angular-momentum-dominated beam production.

The strength and position of the solenoid are selected to get the proper beam transverse size profile, as shown in Fig. 5. Also, as above mentioned, the beam's transverse size reaches its maximum at the entrance of the cavity.

The nonlinear correction results are compared in Table I. Where $\varepsilon_{x, y}$ are emittances of the final flat beam. We can see that $\varepsilon_{x}$ decrease $36 \%$.

In Fig. 6, the left graph is the result of the flat beam with proper correction. The S-shaped distribution has been dramatically straightened. If the beam is overfocused by the solenoid and the transverse size becomes too small somewhere after the compensation solenoid, the resulting nonlinear strong space charge force will produce a reversed Sshaped distribution as shown in the right graph of Fig. 6. This is because the nonlinear space charge force overcorrects the nonlinear if force from the rf cavity.

\section{COMPARE WITH EMITTANCE COMPENSATION}

In the conventional emittance compensation mechanism [12], space charge force is used to align longitudinal slices in phase space to get smaller total effective emittance. In flat beam nonlinear correction, the nonlinear space charge force is used to align different layers in phase space.

Piot [10] has noticed the influence from the spurious momentum to the HTER beam. Smaller momentum spread benefits the matching between the adaptor and the angularmomentum-dominated beam. For the bunch accelerated by 

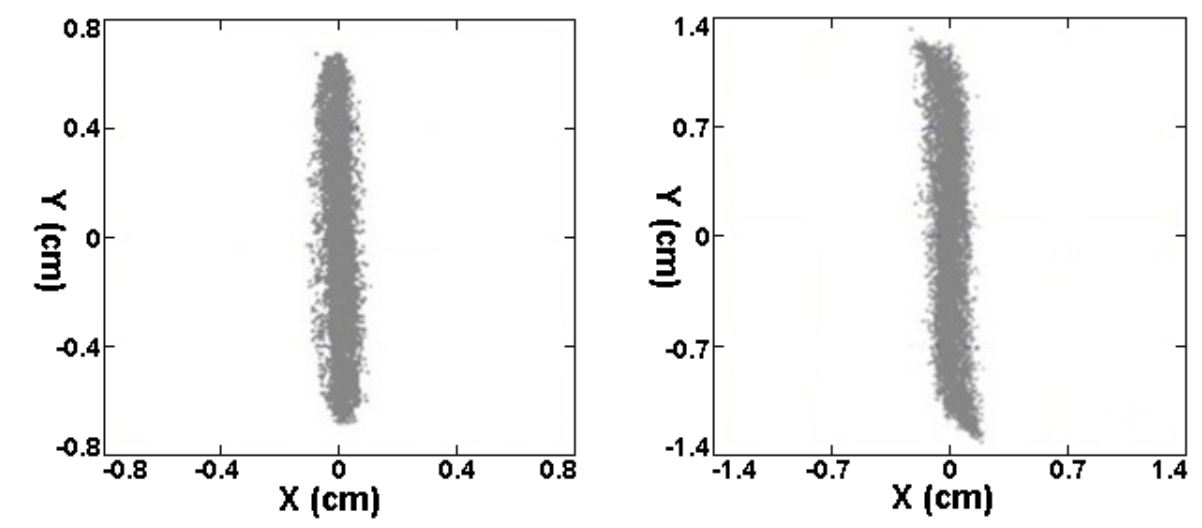

FIG. 6. Nonlinear correction results. Left: with proper correction. Right: with overcorrection.

the rf cavity, there is a correlation between the momentum and longitudinal position within the bunch. Thus, each slice requires a slightly different matching condition from the adaptor. Since the adaptor matching condition is fixed, this momentum spread along the bunch also increases the emittance of the final HTER beam.

\section{CONCLUSIONS}

For the production of HTER beam with a LINAC, a nonlinear correction mechanism is proposed according to the understanding of the source of the S-shaped distribution observed in experimental and simulation results. The nonlinear focusing/defocusing forces are identified as the sources of the S-shaped distribution. With the proposed method, simulations have shown a significantly lower emittance.

\section{ACKNOWLEDGMENTS}

The author would like to thank S. Y. Lee, Don Edwards, Helen Edwards, Steve Lidia, Philippe Piot, and Yin-e Sun for useful discussions. Thanks are also extended to the A0 group at FNAL.
[1] R. Brinkmann, Ya. Derbenev, and K. Flöttmann, Technical report, TESLA Note 99-09, 1999.

[2] A. Zholents et al., Nucl. Instrum. Methods Phys. Res., Sect. A 425, 385 (1999).

[3] S. J. Smith and E. M. Purcell, Phys. Rev. 92, 1069 (1953).

[4] K. Flottmann, R. Brinkmann, and Ya. Derbenev, Phys. Rev. ST Accel. Beams 4, 053501 (2001).

[5] A. Burov, S. Nagaitsev, and Ya. Derbenev, Phys. Rev. E 66, 016503 (2002).

[6] Kwang-Je Kim, Phys. Rev. ST Accel. Beams 6, 104002 (2003).

[7] D. Edwards et al., in Proceedings of the XX International Linac Conference, 2000, p. 122.

[8] D. Edwards et al., in Proceedings of the Particle Accelerator Conference, 2001.

[9] Y.-E Sun et al., Phys. Rev. ST Accel. Beams 7, 123501 (2004).

[10] P. Piot, Y.-E Sun, and K.-J. Kim, Phys. Rev. ST Accel. Beams 9, 031001 (2006).

[11] Lloyd M. Young et al., PARMELA, http://laacg1.lanl.gov/ laacg/services/services.phtml, 2004.

[12] B. E. Carlsten, Nucl. Instrum. Methods Phys. Res., Sect. A 285, 313 (1989). 